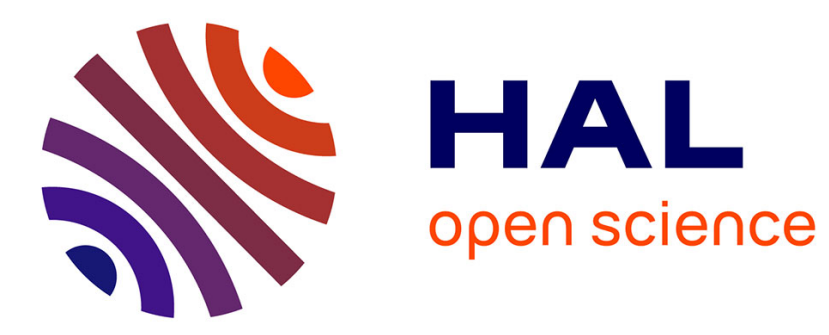

\title{
Energetic Wave Equation for Diffuse Sound Fields
}

Jean-Dominique Polack, Hugo Dujourdy, Baptiste Pialot, Thomas

Toulemonde

\section{To cite this version:}

Jean-Dominique Polack, Hugo Dujourdy, Baptiste Pialot, Thomas Toulemonde. Energetic Wave Equation for Diffuse Sound Fields. Euronoise, May 2018, Heraklion, Greece. hal-01949928

\section{HAL Id: hal-01949928 \\ https://hal.sorbonne-universite.fr/hal-01949928}

Submitted on 19 Dec 2018

HAL is a multi-disciplinary open access archive for the deposit and dissemination of scientific research documents, whether they are published or not. The documents may come from teaching and research institutions in France or abroad, or from public or private research centers.
L'archive ouverte pluridisciplinaire HAL, est destinée au dépôt et à la diffusion de documents scientifiques de niveau recherche, publiés ou non, émanant des établissements d'enseignement et de recherche français ou étrangers, des laboratoires publics ou privés. 


\title{
Energetic Wave Equation for Diffuse Sound Fields
}

\author{
Jean-Dominique Polack \\ Institut Jean Le Rond d'Alembert, Sorbonne Université \& CNRS, Paris, France. \\ Hugo Dujourdy \\ LAUM, Université du Maine \& CNRS, Le Mans, France. \\ Baptiste Pialot \\ Institut Langevin, Paris, France \\ Thomas Toulemonde \\ Impedance, Paris, France.
}

\begin{abstract}
Summary
The diffusion equation for modelling diffuse sound fields was proposed some fifty years ago on heuristic principles as an extension to Sabine's diffuse field model, and still receives much attention. Recently, the authors developed the model one step further to provide the missing relations between sound intensity and sound energy. This introduces some extra terms that, in case of non-Sabine spaces (narrow or flat rooms), can be defined with the help of the boundary conditions in terms of absorption and scattering coefficients on the walls. Integrating the divergence of the stress-energy tensor across the shortest dimensions of the space leads to a propagation equation of the Telegraph type, which can be solved using finite difference time domain simulation. For the two-dimensional case (open-space), numerical results are compared to measurements in real spaces. The comparison makes it possible to evaluate the absorption and scattering coefficients by an adjustment procedure.
\end{abstract}

PACS no. 43.20.Bi, 43.55.Br, 43.55.Cs, 43.55.Dt

\section{Introduction}

Since the first empirical treatment of reverberation by W.C. Sabine in 1894 [1], many authors have noted discrepancies between theory and measurements. But few have questioned the very foundation of Sabine's model, the so-called diffuse field approximation. Among those, ISO 14257 [2] recommends measuring the decay of the sound field with distance, not reverberation time, to characterize industrial halls Ollendorff [3] was the first to come up with the idea of a diffusive model for non-uniform distribution of sound energy in disproportionate rooms. His model escaped attention before being rediscovered independently 20 years later by Picaut et al. [4].

Here we present a new approach based on the stressenergy tensor. Like the diffusion equation [5], this approach was initiated by P.M. Morse [6]. It allows us to write the conservation of sound energy in the form of a Telegraph equation, which is solved by a finite difference method. We then compare the resulting simu-

(c) European Acoustics Association lation to actual measurements in an open-space office. This approach has constituted the doctoral research project of the second author [7], and the topic of two publications $[8,9]$.

\section{State of the art}

\subsection{Sabine's model}

Sabine's model considers the conservation of sound energy at any position in space:

$$
\partial_{t} w+\vec{\nabla} \cdot \vec{I}=0
$$

where $\partial_{t}$ is the partial derivation relative to time, $\vec{\nabla}$. is the divergence operator, $w$ the total instantaneous density of acoustic energy, and $\vec{I}$ the acoustic intensity vector. It adds a heuristic relationship between intensity and sound energy, based on the diffuse field hypothesis:

- sound energy is equi-distributed in space;

- it propagates isotropically in space.

The relationship that binds energy and intensity is then:

$$
|\vec{I}|=\frac{w}{4 \pi} c
$$


where $c$ is the speed of sound; and the total energy incident on the walls with total surface $S$ is given by:

$$
\int_{S} \vec{I} \cdot \vec{n} d S=\frac{w}{4} c S
$$

Taking into account the fact that the acoustic energy is constant in the volume, the integration of the equation over the whole volume $V$ gives the well-known equation:

$$
V \partial_{t} w+\frac{\bar{\alpha} S c}{4} w=0
$$

whose solution is an exponential function of time with decrement $\frac{\bar{\alpha} S c}{4 V}$, where $\bar{\alpha}$ is the average absorption coefficient on the walls.

\subsection{Ollendorff-Picaut's model}

Following a suggestion by Morse and Feshback [5], Ollendorf and Picaut proposed another heuristic relationship between acoustic intensity and energy:

$$
\vec{I}=-\delta \vec{\nabla} w
$$

where the total energy is no longer equi-distributed in space. Here, $\vec{\nabla}$ is the gradient operator and $\delta$ is the diffusion coefficient. They then get an equation of diffusion:

$$
\partial_{t} w-\delta \Delta w=0
$$

or by "renormalizing" the absorption within the volume:

$$
\partial_{t} w+\sigma w-\delta \Delta w=0
$$

This equation accepts an analytical solution in infinite space:

$$
w(\vec{r}, t)=\frac{1}{(4 \pi \delta t)^{\frac{d}{2}}} \exp \left\{-\frac{r^{2}}{4 \delta t}-\sigma t\right\}
$$

where $d$ is the space dimension. In flat spaces $(d=2)$ or narrow spaces $(d=1)$, it can be shown that $\sigma=$ $\frac{\bar{\alpha} S c}{4 V}$, and solution 8 generalizes Sabine's exponential decay.

\section{The stress-energy tensor}

\subsection{General equation}

Following the example of Morse and Ingard [6], Dujourdy et al. $[8,9]$ propose to introduce the symmetric stress-energy tensor:

$$
\underline{\underline{T}}=\left(\begin{array}{cccc}
E & E_{t x} & E_{t y} & E_{t z} \\
E_{t x} & E_{x x} & E_{x y} & E_{x z} \\
E_{t y} & E_{x y} & E_{y y} & E_{y z} \\
E_{t z} & E_{x z} & E_{y z} & E_{z z}
\end{array}\right)=\left(\begin{array}{cc}
E & \vec{J} T \\
\vec{J} & \underline{\underline{E}}
\end{array}\right)
$$

where $\vec{J}=\frac{\vec{I}}{c}$ and $\vec{J}^{T}$ is the transposed matrix of $\vec{J}$; $\underline{E}$ is the wave-stress tensor, with diagonal elements satisfying relation $E=E_{x x}+E_{y y}+E_{z z}$ since kinetic and potential energy are equal on average. The conservation of the tensor $\underline{\underline{T}}$ is simply expressed by a null covariant derivative:

$$
\vec{\nabla}_{4} \cdot \underline{\underline{T}}=0
$$

where $\vec{\nabla}_{4}=\left(\frac{1}{c} \partial_{t}, \vec{\nabla}\right)$ is the quadri-divergence operator.

\subsection{Dimensional reduction}

The dimensional reduction of the system is achieved by integrating on the small dimensions of the volume. Thus, for a flat space such as an open-space office, one integrates on the height of the space (coordinated $z$ ), by introducing the average value of the quantities, except those for which the derivation is carried out according to $z$ and which give the flows entering the walls. For the conservation of energy (first line of the stress-energy tensor), we obtain:

$$
\frac{1}{c} \partial_{t} E l_{z}+\partial_{x} J_{x} l_{z}+\partial_{y} J_{y} l_{z}+J_{z}^{+}-J_{z}^{-}=0
$$

where $l_{z}$ is the height of the space, and where $J_{z}^{+}$, resp. $-J_{z}^{-}$, is the energy flow through the ceiling (side + ), resp. through the floor (side -), i.e. the sound intensity absorbed by each of them.

Similarly, for the following two lines of the stressenergy tensor, the following conservation relationships are obtained:

$$
\begin{aligned}
\frac{1}{c} \partial_{t} J_{x} l_{z}+\partial_{x} E_{x x} l_{z} & +\partial_{y} E_{x y} l_{z} \\
& +E_{x z}^{+}-E_{x z}^{-}=0 \\
\frac{1}{c} \partial_{t} J_{y} l_{z}+\partial_{x} E_{y x} l_{z} & +\partial_{y} E_{y y} l_{z} \\
& +E_{y z}^{+}-E_{y z}^{-}=0
\end{aligned}
$$

where $E_{x z}^{+}$and $E_{y z}^{+}$, resp. $-E_{x z}^{-}$and $-E_{y z}^{-}$, are the wave stresses that are applied on the ceiling $($ side + ), resp. on the floor (side -). It should be noted that the last line of the stress-energy tensor does not provide additional information.

The dimensional reduction is also accompanied by a simplification of the residual wave-stress tensor $\underline{\underline{E}}$, which remains symmetrical. We postulate:

$$
\begin{aligned}
& E_{x x}=E_{y y}=\frac{E}{2} \\
& E_{x y}=0
\end{aligned}
$$

that is, an isotropic distribution of energy in the twodimensional space. 


\subsection{Boundary conditions}

There remains to write the energy balance on the ceiling and the floor of the space, and more generally on the walls. To do this, we introduce the energy distribution function $f(\vec{r}, \vec{v}, t)$, which gives the probability that a wave is localized at position $\vec{r}$ heading in the direction of velocity $\vec{v}$ at instant $t$. Following the example of $[5,4]$, we define $f$ by:

$$
f(\vec{r}, \vec{v}, t)=\frac{E}{4 \pi}+\frac{3 \vec{J}}{4 \pi} \cdot \frac{\vec{v}}{c}
$$

The energy incident on a wall is obtained by integrating on the solid angle $\Omega=(\theta, \phi)$ with $\theta \in\left[0, \frac{\pi}{2}\right]$, $\phi \in[0,2 \pi]$, and the energy reflected by integration on the solid angle $\Omega^{\prime}=(\theta, \phi)$ with $\theta \in\left[\frac{\pi}{2}, \pi\right], \phi \in[0,2 \pi]$. All calculations done, we get:

$$
J_{z, i n c}=\frac{E}{4}+\frac{J_{z}}{2} J_{z, \text { ref }}=\frac{E}{4}-\frac{J_{z}}{2}
$$

and we recover Jing and Xiang's definition of the absorption coefficient $A[10]$ :

$$
\begin{aligned}
J_{z, \text { inc }}-J_{z, r e f}=J_{z, a b s} & =\alpha J_{z, \text { inc }} \\
& =\frac{\alpha}{2(2-\alpha)} E=\frac{A}{4} E
\end{aligned}
$$

where $\alpha$ is a proportionality factor between absorbed and incident intensities, equivalent to an absorption coefficient.

For the stress balance on the walls, it is necessary to add terms to the energy distribution function:

$$
\begin{gathered}
f(\vec{r}, \vec{v}, t)=\frac{E}{4 \pi}+\frac{3 \vec{J}}{4 \pi} \cdot \frac{\vec{v}}{c} \\
+\frac{15}{4 \pi}\left\{E_{x z} \cos \theta \sin \theta \cos \phi+E_{y z} \cos \theta \sin \theta \sin \phi\right\}
\end{gathered}
$$

For the second line of the stress-energy tensor, the incoming and outgoing stresses are obtained by integration in the form of:

$$
\begin{aligned}
M_{x z, \text { in }} & =\frac{3}{16} J_{x}+\frac{1}{2} E_{x z} \\
M_{x z, \text { out }} & =\frac{3}{16} J_{x}-\frac{1}{2} E_{x z}
\end{aligned}
$$

This allows the introduction of a scattering coefficient $D$ :

$$
\begin{aligned}
M_{x z, \text { in }}-M_{x z, \text { out }} & =M_{x z, \text { scat }}=\beta M_{x z, \text { in }} \\
& =\frac{3}{4} \frac{\beta}{2(2-\beta)} J_{x}=\frac{D}{4} J_{x}
\end{aligned}
$$

where $\beta$ is a proportionality factor between scattered and incoming stresses. Similarly, the third line of the stress-energy tensor becomes:

$$
\begin{aligned}
M_{y z, \text { in }}-M_{y z, \text { out }} & =M_{y z, \text { scat }} \\
& =\frac{3}{4} \frac{\beta}{2(2-\beta)} J_{y}=\frac{D}{4} J_{y}
\end{aligned}
$$

\section{Solution}

The substitution of equations 19, 23, and 24 in equations 11-14, as well as the use of the mean free path ( $\lambda=2 l_{z}$ in a 2 -dimensional space), leads to the equation system:

$$
\begin{aligned}
& \frac{1}{c} \partial_{t} E+\partial_{x} J_{x}+\partial_{y} J_{y}+\frac{A}{\lambda} E=0 \\
& \frac{1}{c} \partial_{t} J_{x}+\partial_{x} E_{x x}+\partial_{y} E_{x y}+\frac{D}{\lambda} J_{x}=0 \\
& \frac{1}{c} \partial_{t} J_{y}+\partial_{x} E_{y x}+\partial_{y} E_{y y}+\frac{D}{\lambda} J_{y}=0
\end{aligned}
$$

which reduces to the Telegraph equation:

$$
\frac{1}{c^{2}} \partial_{t t} E-\Delta \frac{E}{2}+\frac{A+D}{\lambda c} \partial_{t} E+\frac{A D}{\lambda^{2}} E=0
$$

\subsection{Finite difference time domain simulation}

We choose to solve numerically equation 25 by a numerical Finite Difference Time Domain (FDTD) method. For this, we choose a non staggered grid and a centred-time centred-space scheme. The derivatives are approximated by:

$$
\begin{aligned}
\left.\frac{\partial^{2} E}{\partial t^{2}}\right|_{x, y} ^{t} & =\frac{E_{i, j}^{n+1}-2 E_{i, j}^{n}+E_{i, j}^{n-1}}{\Delta t^{2}}+\mathcal{O}(\Delta t)^{2} \\
\left.\frac{\partial^{2} E}{\partial x^{2}}\right|_{x, y} ^{t} & =\frac{E_{i+1, j}^{n}-2 E_{i, j}^{n}+E_{i-1, j}^{n}}{\Delta x^{2}}+\mathcal{O}(\Delta x)^{2} \\
\left.\frac{\partial^{2} E}{\partial y^{2}}\right|_{x, y} ^{t} & =\frac{E_{i, j+1}^{n}-2 E_{i, j}^{n}+E_{i, j-1}^{n}}{\Delta y^{2}}+\mathcal{O}(\Delta y)^{2} \\
\left.\frac{\partial E}{\partial t}\right|_{x, y} ^{t} & =\frac{E_{i, j}^{n+1}-E_{i, j}^{n-1}}{2 \Delta t}+\mathcal{O}(\Delta t)^{2} \\
\left.\frac{\partial E}{\partial x}\right|_{x, y} ^{t} & =\frac{E_{i+1, j}^{n}-E_{i-1, j}^{n}}{2 \Delta x}+\mathcal{O}(\Delta x) \\
\left.\frac{\partial E}{\partial y}\right|_{x, y} ^{t} & =\frac{E_{i, j+1}^{n}-E_{i, j-1}^{n}}{2 \Delta y}+\mathcal{O}(\Delta y)
\end{aligned}
$$

where $\mathcal{O}(\cdot)$ is the truncation error and the $\Delta x, \Delta y$ and $\Delta t$ the discretization steps in space and time. The discrete equation then takes the form:

$$
\begin{aligned}
E_{i, j}^{n+1}(a+1) & =E_{i, j}^{n-1}(a-1) \\
& +E_{i, j}^{n}\left(2-C_{r_{x}}^{2}-C_{r_{y}}^{2}-b\right) \\
& +\frac{1}{2} C_{r_{x}}^{2}\left(E_{i+1, j}^{n}+E_{i-1, j}^{n}\right) \\
& +\frac{1}{2} C_{r_{y}}^{2}\left(E_{i, j+1}^{n}+E_{i, j-1}^{n}\right) \\
& +\mathcal{O}\left[(\Delta t)^{2},(\Delta x)^{2},(\Delta y)^{2}\right]
\end{aligned}
$$

completed by 4 boundary equations on the 4 lateral walls of the space [9]. $C_{r_{x}}=\frac{c \Delta t}{\Delta x}$ and $C_{r_{y}}=\frac{c \Delta t}{\Delta y}$ are the Courant-Friedrichs-Lewy coefficients for each dimension, and $a=(A+D) \frac{c \Delta t}{2 \lambda}, b=A D\left(\frac{c \Delta t}{\lambda}\right)^{2}$. The scheme is explicit, and the accuracy is second order in time and space. 


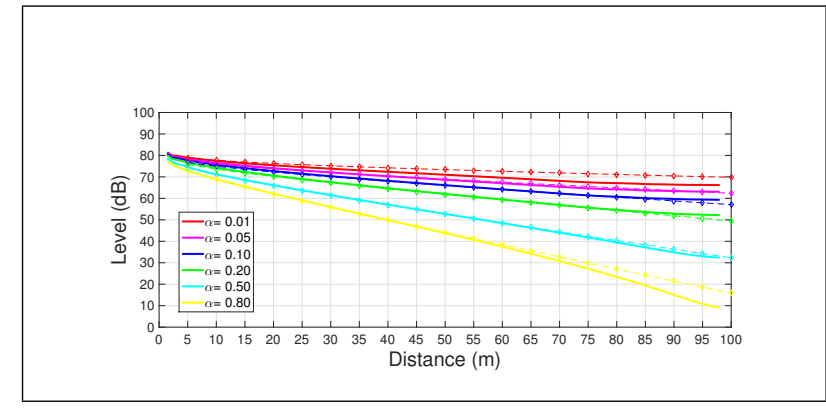

Figure 1. Spatial decays for different $\alpha$ with $\beta=0.2$; lozenges correspond to steady-state analytical solution, with $E_{0}=74 \mathrm{~dB}$

\subsection{Stability}

We study the stability of the scheme with the Von Neumann procedure, which looks for a solution of equation 28 in the form $E_{i, j}^{n}=Z^{n} e^{\mathbf{j}(\theta i+\omega j)}$. All calculations made, we obtain two conditions of stability [9]:

$$
\begin{aligned}
& C_{r_{x}}^{2}\left(1+A D\left(\frac{\Delta x}{2 \lambda}\right)^{2}\right)<1 \\
& C_{r_{y}}^{2}\left(1+A D\left(\frac{\Delta y}{2 \lambda}\right)^{2}\right)<1
\end{aligned}
$$

\section{Results}

The discrete equation 28 was applied to a quasiinfinite two-dimensional space of $200 \mathrm{~m}$ by $200 \mathrm{~m}$. The source is located in the centre of the space. The absorption and scattering coefficients range from $\alpha=$ 0.01 to $\alpha=0.8$ and $\beta=0.01$ to $\beta=1.5$. The mean free path is $\lambda=5.4 \mathrm{~m}$, and the discretization steps are set at $\Delta x=\Delta y=1 \mathrm{~m}$ and $\Delta t=1 \cdot 10^{-3} \mathrm{~s}$. The speed of sound is $c=344 \mathrm{~m} / \mathrm{s}$.

\subsection{Spatial decay}

Figures 1-2 show spatial sound decays, and compare them to the analytical solution in the steady-state case:

$$
E=E_{0} \frac{\exp \left(-\frac{\sqrt{2 A D}}{\lambda} r\right)}{\sqrt{r}}
$$

for large values of $r$, where $r$ is the distance to the source and $E_{0}$ an integration constant.

It is clear that the numerical solution corresponds to the analytical solution, except for the very low values of the coefficients, or near the walls where reflections on the latter exert influence.

\subsection{Temporal decay}

Figures 3-4 show temporal sound decays at $15 \mathrm{~m}$ from the source, and compare them to the two analytical approximations corresponding resp. to a large value of one of the coefficients and to equal coefficients. In

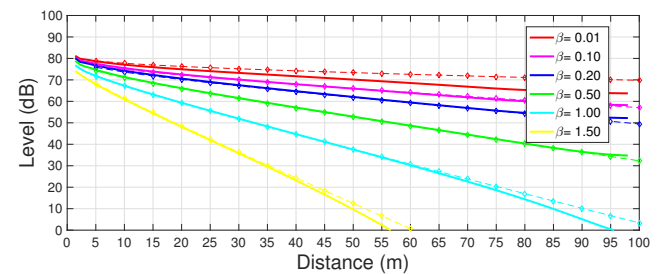

Figure 2. Spatial decays for different $\beta$ with $\alpha=0.2$; lozenges correspond to steady-state analytical solution, with $E_{0}=74 \mathrm{~dB}$.

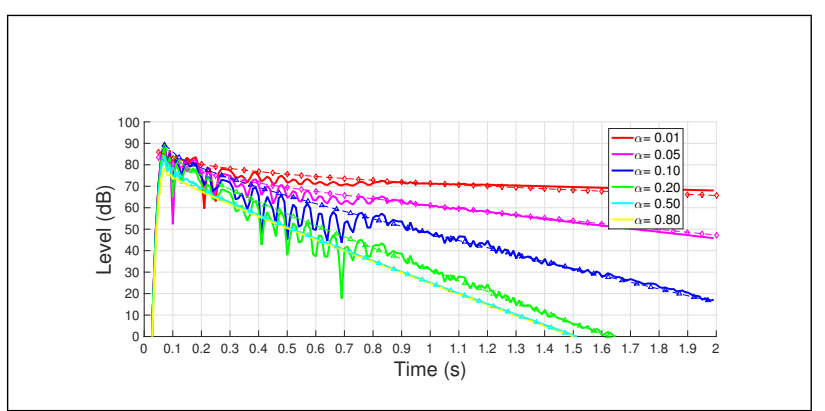

Figure 3. Time decays at $15 \mathrm{~m}$ from source for different $\alpha$ with $\beta=0.2$; lozenges correspond to solution 31 and triangles to solution 32 .

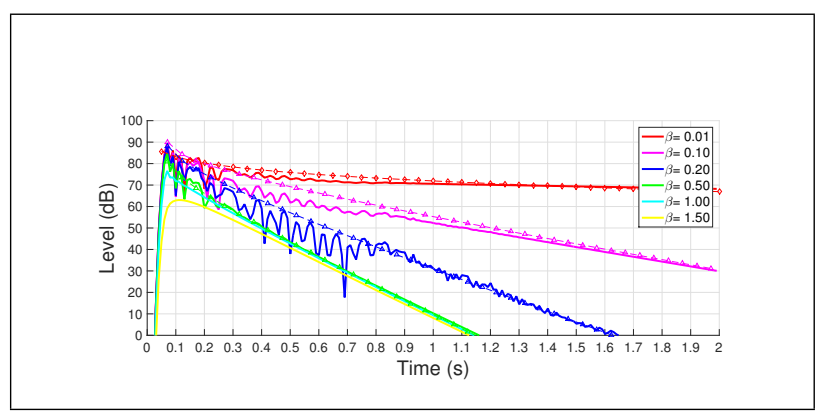

Figure 4. Time decay at $15 \mathrm{~m}$ from source for different $\beta$ with $\alpha=0.2$; lozenges correspond to solution 31 and triangles to solution 32 .

the first case, equation 25 is reduced to an equation of diffusion, with solution:

$$
E=E_{1} \frac{e^{\left(-\frac{(A+D) r^{2}}{2 \lambda c t}-\frac{A D c}{(A+D) \lambda} t\right)}}{t}
$$

In the second case $(A=D)$, the solution is a travelling wave packet [11]:

$$
E^{+}=E_{2} e^{\left(-\frac{A}{\lambda} c t\right)} \frac{U\left(\frac{c}{\sqrt{2}} t-r\right)}{2 \pi \sqrt{\left(\frac{c}{\sqrt{2}} t\right)^{2}-r^{2}}}
$$

where $U(\cdot)$ is the Heaviside function.

It is clear that the numerical solution corresponds to the analytical solutions with appropriate choice of the integration constants $E_{1}$ and $E_{2}$, especially for the 


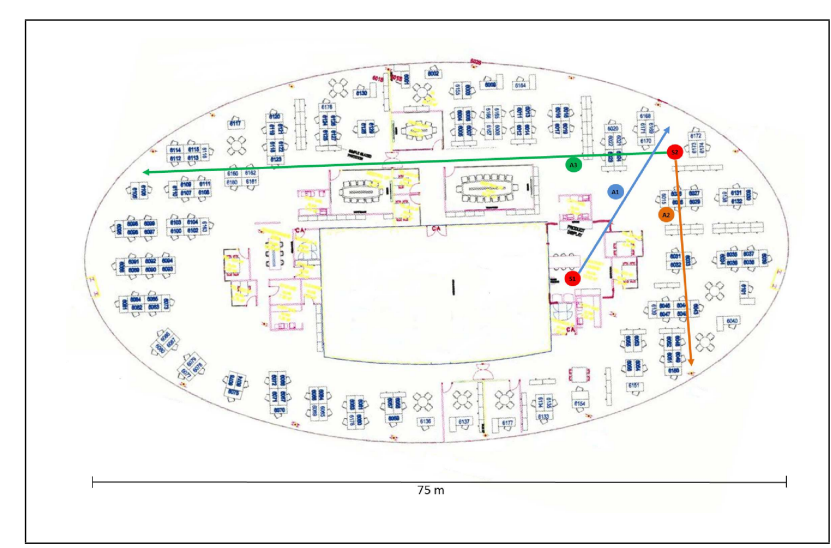

Figure 5. Plan of Ovalie: measurements use S3 source and follow green axis.

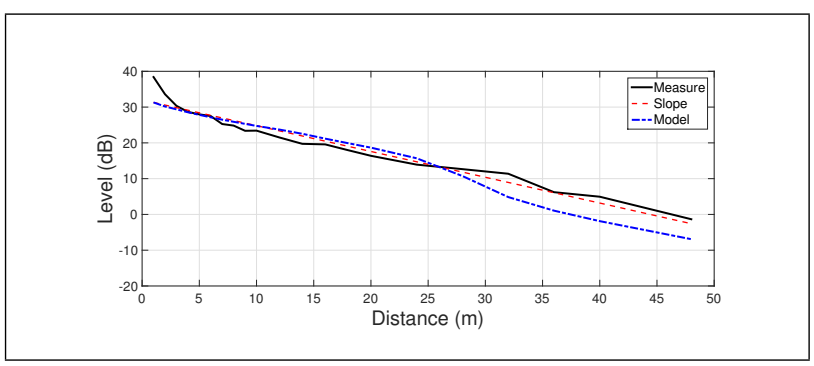

Figure 6. Model adjustment by comparison with spatial decay measured at Ovalie: source S3, green axis, $\alpha=0.21$ and $\beta=0.7$.

large values of $t$ when the algorithm has stabilized. In Figures $3-4, E_{1}=74 \mathrm{~dB}$ for analytical solution 31 ; for analytical solution $32, E_{2}=101 \mathrm{~dB}$, but reduces to $95 \mathrm{~dB}$ for large values of $\alpha$ and $\beta$. Similar results are obtained at the other distances.

\subsection{Measurements}

We were able to conduct measurements in three openspace offices in Paris. Here we present the results obtained for Ovalie (Figure 5), an office space of $75 \mathrm{~m}$ long by $35 \mathrm{~m}$ wide, oval-shaped with a central islet. Its surface is $1260 \mathrm{~m}^{2}$, and its height $2.7 \mathrm{~m}$, yielding a mean free path of $5.4 \mathrm{~m}$. The office was furnished with desks, chairs and computers during measurements, but without the presence of users.

Figure 6 shows the spatial decay in Ovalie along the green axis and Figure 7 the temporal decay at $10 \mathrm{~m}$ from the S3 source. Both Figures compare measured decays to the decays calculated after adjusting the parameters $\alpha$ and $\beta$. The measurement frequency is the $1 \mathrm{kHz}$ octave, and the optimum values of $\alpha$ and $\beta$ are respectively 0.21 and 0.7 . The same optimum values are obtained at the other distances.

In Figure 7, the levels of the temporal decay are slightly overestimated, an observation that is general to all analysed positions.

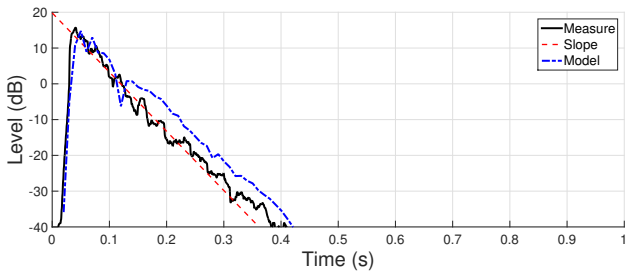

Figure 7. Model adjustment by comparison with temporal decay at $10 \mathrm{~m}$ from the source measured at Ovalie: source S3, green axis, $\alpha=0.21$ and $\beta=0.7$.

\subsection{Adjustment procedure}

The absorption and scattering coefficients are obtained by comparison with the measurements. Now we see in Figures 3-4 that the smaller of the $\alpha$ or $\beta$ values drives the temporal decay. In other words, temporal decay allows the evaluation of the smaller of the coefficients, in general $\alpha$, and the second coefficient is obtained from spatial decay. Thus an iterative procedure was used to estimate $\alpha$ and $\beta$, assuming first $\beta$ large for a first estimation of the absorption coefficient by equation 31 , which reduces to:

$$
E \approx E_{1} \frac{e^{\left(-\frac{(D) r^{2}}{2 \lambda c t}-\frac{A c}{\lambda} t\right)}}{t}
$$

Then $\beta$ is inferred from the spatial decay by equation 30 . The procedure however exaggerates the slopes of the two decays, so that the coefficients are slightly adjusted to the margin for better agreements with the measures.

\section{Conclusions}

We have shown that energy conservation can be broken down into two equations: the conservation of total energy; and the conservation of the acoustic intensity. The two equations combine into a single tensor equation, the conservation of the stress-energy tensor. The resolution of these equations involves two coefficients on the walls: an absorption coefficient and a scattering coefficient. The smaller of these two coefficients, usually the absorption coefficient, pilots the temporal decay, and the second coefficient is then obtained from spatial decay. This adjustment procedure has been validated in real cases of open-space offices.

\section{Acknowledgement}

This work was carried out within the framework of a CIFRE convention ( $\mathrm{nr}$ 2012/1184) between Impedance S.A.S. and Université Pierre et Marie Curie. The authors thank Impedance for providing access to the open-space offices for the measurements.

\section{References}


[1] W. C. Sabine: Collected papers on acoustics. Peninsula Publishing, 1993.

[2] Acoustics - Measurement and parametric description of spatial sound distribution curves in workrooms for evaluation of their acoustical performance. ISO Standard 14257, 2001.

[3] F. Ollendorff: Statistical room acoustics as a problem of diffusion: a proposal. Acustica 21 (1969) 236-245.

[4] J. Picaut, L. Simon, J.D. Polack: A mathematical model of diffuse sound field based on a diffusion equation. Acta Acust united Ac 83 (1997) 614-621.

[5] P. M. Morse, H. Feshbach: Methods of Theoretical Physics. McGraw-Hill, 1953.

[6] P. M. Morse, K. U. Ingard: Theoretical Acoustics. McGraw-Hill, 1968.

[7] H. Dujourdy: Diffusion acoustique sur les lieux de travail. PhD Thesis, UPMC, 2016. Online at: http://www.lam.jussieu.fr/Publications/Theses/thesehugo-dujourdy.pdf.

[8] H. Dujourdy, B. Pialot, T. Toulemonde, J.D. Polack: An energetic wave equation for modelling diffuse sound fields - application to corridors. Acta Acust united Ac 103 (2017) 480-491.

[9] H. Dujourdy, B. Pialot, T. Toulemonde, J.D. Polack: An energetic wave equation for modelling diffuse sound fields - application to open offices. Submitted to Wave Motion (2018).

[10] Y. Jing, N. Xiang: On boundary conditions for the diffusion equation in room acoustic prediction: theory, simulation, and experiments, J. Acoust. Soc. Am. 123 (2008) 145-153.

[11] M. Bruneau: Introduction aux théories de l'acoustique. Université du Maine, 1983. 